\title{
Lexicografía misionera bilingüe novohispana: el vocabulario náhuatl-castellano de 1571
}

\author{
Missionary Bilingual Lexicography in New Spain: \\ the Vocabulary List in Nahuatl-Castilian of 1571
}

\author{
Manuel Galeote \\ Universidad de Málaga \\ galeote@uma.es \\ Miguel Figueroa-Saavedra \\ Universidad Veracruzana (México) \\ migfigueroa@uv.mx
}

Resumen

Con el Vocabvlario en lengva mexicana y castellana (México, 1571) culminan los trabajos lexicográficos sobre la lengua náhuatl del equipo de misioneros franciscanos, con Alonso de Molina a la cabeza desde 1555, impulsados por fray Bernardino de Sahagún. Anteriormente, ya Andrés de Olmos había reunido un corpus de voces autorizadas a partir de textos para lograr un vocabulario completo. El paradigma franciscano siguió la senda de Olmos, pero perfiló los matices del significado y uso de las voces glosadas. Los misioneros tenían la urgencia de componer discursos (sermones, pláticas) y difundir documentos escritos, correctos y respetuosos con las normas gramaticales del náhuatl. Los dos vocabularios castellano-náhuatl (1555-1571) fueron ensayos previos de Molina. Se separaban cualitativa y cuantitativamente de lo que nos ha llegado de Olmos. Pero en este Vocabulario mexicanocastellano, Molina demostró la altura de su talla de lexicógrafo y de gramático, pues también en el mismo año 1571 publicó el Arte de la lengua mexicana. Desde el punto de vista de la microestructura lexicográfica, fray Alonso proporcionó información sobre la construcción del verbo, así como también hacía con el sustantivo o el adverbio. En el orden alfabético, los verbos mostraban los prefijos y la modificación de la raíz en la forma del perfecto. Lograda una ordenación alfabética de las entradas más práctica, la búsqueda y la integración de las características morfosintácticas de la lengua aglutinante resultó más fácil. El final del histórico proyecto comunitario franciscano fue imprimir aquella obra lexicográfica, concebida para "provecho del prójimo", que contenía mucha información gramatical, le dotaba de utilidad didáctica y traductora, y superaba a todas por "mejor declarar cada vocablo de la lengua" náhuatl.

Palabras Clave: lexicografía, lingüística misionera; vocabulario bilingüe, lengua náhuatl, Nueva España, Alonso de Molina 
Abstract

The lexicographical works of the Franciscan missionary team is culminated with the Vocabvlario en lengva mexicana y castellana (Mexico, 1571), with Alonso de Molina leading it, and which Bernardino de Sahagún had promoted as early as 1555. Previously, Andrés de Olmos had already put together a corpus of texts by authorized authors to achieve a complete vocabulary of the Nahuatl language. The Franciscan paradigm followed the path of Olmos, but he outlined the nuances of the meaning and use of the glossed words. The missionaries were urged to compose speeches (sermons, pláticas) and to distribute written documents that were correct and respectful following the grammatical norms of Nahuatl. The two Castilian-Nahuatl vocabularies $(1555,1571)$ were previous attempts of Molina. They are qualitatively and quantitatively different from what Olmos produced. But in this Mexican-Spanish vocabulary, Molina demonstrated his ability as a lexicographer and grammarian, especially given his publications of the Arte de la lengua mexicana in the same year 1571. From the point of view of the lexicographical microstructure, fray Alonso explained verb construction as well as he did with noun or adverb. In alphabetical order, the verbs include prefixes and the modification of the root in the perfect form. With a more practical alphabetical ordering of the entries, the search and integration of the morphosyntactic characteristics of the agglutinating language was made easier. The final step of the historic Franciscan project was to print this lexicographical work, conceived as the "provecho del prójimo", which contained an abundance of grammatical information, proving useful as a tool for translation and instruction, surpassing previous work as as "mejor declarar cada vocablo de la lengua" náhuatl.

KeYworDs: lexicography, missionary linguistics, bilingual vocabulary, Nahuatl language, New Spain, Alonso de Molina

FECHA DE RECEPCIÓN: 07/09/2018

FECHA DE ACEPTACIÓN: 07/01/2019

\section{Un magno proyecto editorial franciscano: del manuscrito al impreso}

Con la llegada de la imprenta española a las tierras novohispanas, la lingüística misionera dispuso de una incalculable ayuda para la producción de materiales abundantes (catecismos, confesionarios, artes y diccionarios). Al llegar el último tercio del siglo XVI, la imprenta de Antonio de Espinosa dio a la estampa un volumen doble de fray Alonso de Molina, cuya segunda parte 
se titulaba Vocabvlario en lengva mexicana y castellana (1571). ${ }^{1}$ Las páginas que siguen mostrarán un análisis minucioso de los datos de que disponemos sobre la Historiografía lingüística misionera de tradición hispánica en México, apoyada en las fuentes originales. Además, se expondrá con razones argumentativas el desarrollo interlingüístico (castellano-náhuatl) que entre 1555 y 1571, a la sombra de Sahagún y de Olmos, convirtió a fray Alonso de Molina en el misionero más relevante que imaginar se pudiera en la elaboración de un vocabulario bidireccional náhuatl-castellano.

Para el taller editorial franciscano, ${ }^{2}$ la publicación supuso un hito en la lexicografía novohispana. La imprenta de Antonio de Espinosa produjo una obra de gran alcance. El autor franciscano le había reportado buenos beneficios con la publicación de confesionarios. El libro contribuyó "mucho a la fama de nuestro impresor, y del cual se han conservado numerosos ejemplares [...]. Sólo superado en 1885 por el Dictionnaire de la Langue nauatl ou mexicaine, de Rémi Siméon, publicado en París por la Imprenta National" (Stols, 1989: 16). La edición impresa requirió mucho tiempo por el volumen y composición de tipos en lengua náhuatl. Aproximadamente, se tuvieron que componer 24,000 entradas en náhuatl en 325 páginas, lo que supone un trabajo de gran envergadura, pues el Vocabulario parejo de la lengua castellana y mexicana de 1571, se compone de sólo 242 páginas, sumando incluso la parte dedicada a la "cuenta numeral”. Además, la apuesta tipográfica, al renunciar a los tipos en letra gótica, aumentó el trabajo pero también el prestigio, convirtiéndose el doble Vocabulario de 1571 en uno de los más afamados incunables americanos (Lafaye, 2002: 97).

\footnotetext{
${ }^{1}$ Para mayor comodidad del lector, recurriremos en el texto a tres siglas de las obras originales de Molina, según se indica en la bibliografía final: VCM55: Alonso de Molina, Aquí comiença vn vocabulario en la lengua castellana y mexicana, México, Juan Pablos, 1555; VCM71: Vocabvlario en lengva castellana y mexicana, México, Casa de Antonio de Spinosa, 1571; VMC: Vocabvlario en lengva mexicana y castellana, México, Casa de Antonio de Spinosa, 1571.

${ }^{2}$ Véase Hernández y Léon Portilla, 2009: 80-84, con especial atención a los franciscanos en Mesoamérica.
} 
Es bien reconocido que esta obra como corpus lexicográfico es insustituible y básica, tanto por el acopio de vocablos como por la modernidad de su disposición, lo cual ha sido apreciado desde el mismo surgimiento de la lingüística moderna (Siméon, 1977: XXXIII, LXXXVII; León-Portilla, 1977: LX; Ringmacher, 1997: 75 y ss.). Al convertirse el manuscrito en un texto impreso, su difusión y utilidad excedieron los límites de la comunidad franciscana.

\section{Los antecedentes y desarrollo del proyecto lexicográfico}

Este gran esfuerzo editorial no es, sin embargo, más que el punto final de una labor mucho más compleja, en el que destaca el concepto y el método de un diccionario en la lengua náhuatl como lengua de partida. Desde 1530 algunos franciscanos realizaron varios esfuerzos en ese sentido. Fray Francisco Ximénez se destacó por ser el primero que realizó un arte y vocabulario de la lengua mexicana (Mendieta, 1973: II, 118). Y aunque hubo otros frailes que también se mostraron doctos en esta materia, no fue hasta fray Andrés de Olmos cuando existió un nuevo intento de crear un vocabulario náhuatl, al igual que de otras lenguas indígenas (Mendieta, 1973: II, 118). Fechado su manuscrito del Arte de la lengua mexicana en 1547, este arte no se limitaba a describir la gramática de la lengua, sino que contemplaba la necesidad de reunir un corpus lexicográfico amplio que permitiera compilar un vocabulario completo.

Sin embargo, en la macroestructura lexicográfica se hacen evidentes las dificultades estructurales para el vocabulario y su posterior vertebración en un diccionario (Galeote y FigueroaSaavedra, 2010). Si observamos la parte final del Arte de Olmos, se advierte que la ordenación de los vocablos responde a criterios de agrupación meramente descriptivos y muy provisionales. Podría afirmarse que se trata de un esbozo de vocabulario temático y diccionario con materiales de la gramática. ${ }^{3}$ Así, se

\footnotetext{
${ }^{3}$ Sobre los tratados gramaticales novohispanos, véase Hernández y León-Portilla, 2009.
} 
aborda el nombre mediante la búsqueda de equivalentes en náhuatl a diversas expresiones romances de uso general. También se rastrea la expresión en forma elegante y culta de expresiones del náhuatl, sacadas de los huehuètlàtolli. Después de estos capítulos $\left(7^{\circ}\right.$ y $\left.8^{\circ}\right)$, siguen los apuntes sobre diversas cuestiones verbales. Se incluye también una relación de prefijos sujetos y objetos, como esbozo de una relación de expresiones verbales y léxicas en náhuatl (que "son provechosas", Olmos, 1993: 228 r.). Hay textos en náhuatl con apelativos que recibían los dioses; hay fórmulas para la celebración del matrimonio, junto con expresiones verbales y nombres de parentesco y, por último, una relación de verbos en ambas lenguas.

Conviene observar con más detalle estos contenidos porque entenderemos qué perseguía Molina con su Vocabulario. Los capítulos $7^{\circ}$ y $8^{\circ}$ del Arte de Olmos mostraban el deseo de acopio de textos para capturar vocablos dentro del contexto discursivo. Esto no es algo original de Olmos sino que parece una práctica general - la "red barredera" - llevada a su máxima expresión en el proyecto de Calepino de fray Bernardino de Sahagún (Máynez, 2002). Esta relación pretende identificar expresiones retóricas equivalentes que garanticen, por un lado, la comprensión del texto a un potencial oyente, pero también una determinada calidad retórica que salve una de las grandes preocupaciones de los frailes misioneros: no solo ser entendidos, sino también respetados. Así, en 1565 Molina observaba en su Confesionario Mayor "la obscuridad y difficultad de la dicha lengua destos naturales [...] muy differente en muy muchas cosas de nuestra lengua castellana y latina, con las quales midiendola, avria gran differencia y desigualdad (como es manifiesto a los que desta lengua tienen clara noticia)" (1565: f. 2). Lograr el respeto como efecto del principio de autoridad del que habla bien y maneja todos los registros de la lengua es una cuestión cuya preocupación resulta notoria en toda la obra de los misioneros.

En ese sentido, los desvelos por aprender la lengua indoamericana conllevaban la acumulación de textos de los que extraer no sólo vocablos, sino la razón pragmático-semántica que mostraría su significado, con todos los matices denotativos 
y connotativos. Esto posibilitaba, en principio, la composición de nuevos textos, con un modelo sintáctico y gramatical "correcto" en lengua náhuatl. Se daba por sentado que en ocasiones no existiría un equivalente literal para un término específico ni para oraciones o expresiones fraseológicas estereotipadas. A este respecto, el propio Olmos declaraba:

Cosa prolixa seria poner todas las orationes en las que les discrepa nuestro Romance de la manera de decir desta lengua, quiero decir que lo yndio no corresponde al castellano. Pondremos algunas que son comunes y ponerse an por la orden de los aduerbios principales que las tales oraciones se ponen para que mejor se halla la oration que queremos conver $<\mathrm{t}>$ ir en la lengua.

Después pondremos algunos otros Romances estrauagantes y destos que aquí se pusieren ansi de los vnos como de los otros podemos tomar sino para por ellos hacer otras orationes semejante $<\mathrm{s}>$. (1993: f. 199a r.-v.)

El uso de expresiones específicas, que se proponen como modelo idiomático para ser intercaladas en el discurso por los misioneros-estudiantes, en una situación comunicativa dada, se completa con el repertorio "lexicográfico" pragmático que cierra el Arte. Así Olmos incluye advocaciones divinas que pueden aplicarse a Dios y fórmulas rituales para el matrimonio católico, junto con frases hechas que son requeridas por las necesidades comunicativas en diferentes contextos. Sin embargo, se aprecia una tendencia a tomar de esas oraciones aquellos vocablos que son más fáciles de ordenar — sustantivos y verbos-, y cuyo abultado número incrementa el caudal de materiales para la confección de un verdadero diccionario que estableciera los equivalentes semánticos más adecuados junto con los matices y perfiles del significado del lema.

Si este Arte manuscrito de Olmos fue su contribución al análisis gramatical del náhuatl y la base para ser continuada por otros hermanos franciscanos, Molina tuvo que ser la persona más competente para seguir lo que se había quedado en un esbozo: la creación de un verdadero y amplio corpus lexicográfico 
náhuatl. Esto es verosímil, pues el Arte de Andrés de Olmos se da por terminado cuando ejercía de guardián en el Monasterio de San Andrés en Hueytlalpan. Allí centraba entonces sus trabajos en el estudio del idioma totonaca y se preparaba para su incursión en la Huasteca. No podemos afirmar que Olmos ultimara un Vocabulario náhuatl, pues solo conservamos un ensayo, una prueba o un borrador (folios 225-288), cuyo manuscrito se custodia en el Middle American Research Institute de la Universidad de Tulane (Nueva Orleans, Estados Unidos) y fue reproducido facsimilarmente por René Acuña (Olmos, 1985). Aunque la copia manuscrita carece de algunas partes - el capítulo $8^{\circ}$ está incompleto y no aparece el anunciado primer huehuètlàtolli-, destacados especialistas reconocen que el vocabulario que tiene al final es fruto indudable del trabajo de Andrés de Olmos ${ }^{4}$ (Hernández y León Portilla, 1993: xli). En principio, creemos que este manuscrito es una recopilación de materiales, con enfoque semántico y gramatical para extraer formas léxicas, gramaticales, expresiones y estructuras idiomáticas, con marcación sociolingüística y pragmática.

Desde una perspectiva amplia, se llega a inventariar la terminología por campos semánticos (se agrupan los términos de parentesco, por ejemplo). Asimismo, se justifica la ordenación del material por la pertenencia a la categoría gramatical, como en el Arte de Olmos. La mención de un vocabulario terminado por Olmos (Mendieta, 1973: II, 179) no ha podido corroborarse por no haberse hallado (Hernández y León Portilla, 1993: xxxii).

\footnotetext{
${ }^{4}$ Un argumento que podemos añadir a los expuestos por tales expertos a través del análisis comparativo del contenido es el hecho característico, desde un examen caligráfico de la aparición de una peculiar marca disléxica o disortográfica que suele presentarse de vez en cuando en las obras de Olmos, sea de su propia mano o de la fidelidad a la letra de sus copistas y transcriptores (si no una propia característica de su escribano). En el f. 253v. se dice "dar coces" para a continuación repetir esta expresión como "dea coces"; en el f. 262v. de este Vocabulario, aparece la entrada "arredondeca" por "arrendodear", y en el f. 263v. "amargca" por "amargar". Este lapsus calami vuelve a reproducirse en su Tratado de hechicerías y sortilegios de 1553, en cuyo prólogo aparece la palabra "aducasario" por "aduersario", por lo que parece probable que esta alteración de ar/er $>$ ea/ca sea una marca característica de los manuscritos de Olmos.
} 
En consecuencia, aunque no nos hallemos ante el Vocabulario al que se refería Mendieta, no podría ser muy diferente de lo que nos ofrece el manuscrito de Tulane. Por deducción, donde acabó el trabajo de Olmos empezó el de Molina.

Cabe replantearse la magnitud del Vocabvlario que comienza en la llamada entonces lengua mexicana (VMC71) no como logro individual de un autor, sino como la culminación de un vasto proyecto comunitario dilatado en el tiempo; la suma de sucesivos esfuerzos acumulados por el trabajo en equipo que desarrollaron los franciscanos en la Nueva España desde 1530. Hacia 1550, dadas las necesidades evangelizadoras y lingüísticas, los misioneros precisaban un manual con la relación completa, ordenada y clara de todos los vocablos y formas de expresión de la lengua náhuatl. Dicho manual permitiría aprender la lengua y elaborar textos sin la dependencia de plantillas ni fórmulas discursivas. Era necesario desarrollar la competencia lingüística del aprendiz para superar las necesidades comunicativas interlingüísticas. Solo así podrían entablarse conversaciones auténticas, de manera que se difundiera el Evangelio y comprender lo que el oyente entendía. Con estos desarrollos lingüístico-comunicativos, el misionero profundizaba en el estudio superior del náhuatl. Tal dominio de la lengua posibilitaba dirimir dudas religiosas y debatir sobre los conceptos divinos, significados de los vocablos o proponer ejemplos, como muestran claramente los Colloquios de los doce frailes (Anónimo, 1986).

\section{De la lista de vocablos en náhuatl a la microestructura del Vocabulario}

Si partimos de esa continuidad, las preguntas que nos formulamos y que se debió de plantear Molina son las siguientes: 1) ¿hasta qué punto podía tomarse como una herramienta útil el trabajo hecho hasta ahora? 2) ¿en qué medida podía considerarse que era representativo de todo el amplio repertorio de voces nahuas? Y lo más importante, desde una perspectiva pragmalingüística, 3) ¿hasta qué punto este material era aprovechable en las labores misioneras? De la respuesta a la primera pregunta dependen, en 
el mismo sentido, las respuestas siguientes. Sin haber resuelto aquella duda, no se podía plantear el añadir más vocablos a los registrados previamente o la redacción de nuevos textos sin la garantía de disponer de un repertorio de voces y expresiones usuales completo. ${ }^{5}$

Si examinamos los materiales de Olmos, la diversidad de criterios refleja diferentes concepciones que se debaten entre la extrañeza ante una exótica lengua y la fidelidad a unos modelos considerados eficaces. Sospechamos que el modelo que se encontraba en la mente de Olmos para un Vocabulario debía de ser muy parecido al apéndice ofrecido por fray Pedro de Alcalá en su Arte para ligeramente saber la lengua arauiga (1505) para el aprendizaje del árabe, tras la conquista del reino nazarí de Granada. Este Arte se ve complementado por el Vocabulista castellano-árabe, ${ }^{6}$ en el que se ordena un amplio léxico propio del habla común, agrupado y ordenado alfabéticamente por su primera sílaba, siguiendo el modelo nebrisense:

Acorde escojer vna de las co[m]pilaciones que ay de vocablos para la trasladar en arauigo y entre otras pareciome acomodada a nuestro castellano aquella que hizo el honrrado y prudente varon maestro Antonio de Lebrixa, a la qual yo añadi algunos nombres y verbos, y otras partes de la oracion que me ocurrieron y dexe algunas de las que alli estauan que carecian de traslación arauiga. [...] E cuidando que estaua el romance como deuia estar ordenado de mano de tal varon, non cure de mirar en la ordel del proceder mas solamente

\footnotetext{
${ }^{5}$ Véase Montes de Oca Vega (2013 y 2016) para conocer mejor el trasvase de terminología entre lenguas, la neología en náhuatl y los aspectos interlingüísticos de las voces religiosas. Véase también Galeote (en prensa).

${ }^{6}$ La estructura del Arte de Pedro de Alcalá trataba los siguientes aspectos gramaticales: a) el alfabeto y su pronunciación; b) las partes de la oración; c) el nombre (especie, género, número, plurales, figura, casos y declinaciones, diminutivos, nombres derivados de verbos), d) el pronombre (posesivos, declinación, relativo); e) el verbo (accidentes, género, conjugación, modos, tiempos, números, personas, pasiva, impersonal, pretérito, verbos irregulares, imperativo, verbos intrínsecos, infinitivo, participio de pretérito, nombre verbal, gerundio); f) conjunciones; g) preposiciones; h) interjecciones; i) reglas de lectura; y j) oraciones, fórmulas rituales, impartición de sacramentos y doctrina en lengua árabe.
} 
mire a los principios de las partes donde vino que en la orden del abc que suelen leuar los vocabulistas, ay alguna poca turbación (Alcalá, 1505: Prólogo).

Por tanto, la adopción del modelo de Nebrija se hizo de manera flexible de acuerdo con las peculiaridades de la lengua y la traducción:7 "En cada vna de las letras se ponen primero los verbos, y luego los nombres y en fin de los nombres los adverbios, conjunciones y preposiciones" (Alcalá, 1505: Prólogo). Al final del vocabulario se añadió un listado de numerales. Olmos procedía como los evangelizadores del antiguno reino nazarí de Granada: Arte más textos de apoyo para la evangelización y un vocabulario con verbos y nombres. Se esforzó por incluir vocablos y "modos de hablar" elegantes (hablantes cultos). Evidentemente, la ambición del proyecto rebasaba las características de un "arte ligero" o tratado introductorio. Era necesario parcelar las tareas y trabajar independientemente con artes, doctrinas, sermonarios, confesionarios y vocabularios.

Para el vocabulario, Olmos planeó la ordenación por palabras clave y campos semánticos. Las frases hechas del castellano se ordenaban por el adverbio. Las entradas de verbos castellanos iban agrupadas por su letra inicial, alfabéticamente, aunque sin la ordenación alfabética interna. Esto demuestra el carácter de trabajo inacabado.

\subsection{Apectos de la macroestructura gramatical}

El principal problema de los franciscanos se presentaba al elaborar un vocabulario náhuatl-castellano. Era necesario reunir voces, significados y acepciones metafóricas (también ausentes en Vocabulista de Alcalá [Pezzi, 1989: 11]). En principio una clasificación de las palabras por categorías gramaticales exigiría del lector el conocimiento y capacidad de identificación morfológica de las palabras en la lengua náhuatl. El problema desaparecía si todas las entradas se ordenaban alfabéticamente como

\footnotetext{
${ }^{7}$ Véanse las páginas que a Nebrija le dedica desde México Ascensión Hernández (2014: IX-XLV) en la edición de la Gramática que anotó y editó Carmen Lozano.
} 
hizo Nebrija y como acabó haciendo Molina. La macroestructura tuvo que lidiar con las entradas de carácter verbal. Olmos esbozó un diccionario bilingüe de los verbos. No sólo hay una relación de verbos en castellano con su equivalencia en náhuatl, sino una relación de verbos en náhuatl con su equivalencia en castellano. La comparación de sus diferentes modos de ordenación y exposición arroja apreciaciones muy interesantes sobre el proceso de lematización. ${ }^{8}$

Molina fue capaz de llegar más lejos que Olmos y alumbrar el vocabulario náhuatl de 1571, que debería de haberse impreso y encuadernado delante del vocabulario castellano-náhuatl. Pero aquel desafío solo se puede superar en la época actual, cuando los desarrollos de lingüística descriptiva permiten que desplacemos el foco de interés de la lengua española a la lengua autóctona. No creemos que sea casual que la fecha de 4 de mayo en que se databa el colofón de 1555, sea la misma fecha en que Molina firmó la Epístola nuncupatoria de los vocabularios de 1571. ${ }^{9}$ La preocupación de los franciscanos, Molina el primero, era describir el náhuatl: el castellano ya lo sabían los misioneros. Quienes usaban el diccionario eran los misioneros. Por tanto, no se perseguía un diccionario castellano, sino un vocabulario y una gramática del náhuatl.

La compilación de verbos de Olmos se enfrentaba a la sistematización lexicográfica del náhuatl: ¿cómo lematizar una lengua aglutinante? Hoy la respuesta puede parecernos muy fácil: basta con aislar la raíz nominal o verbal de una palabra. No obstante, esa solución planteaba un gran problema a los franciscanos pues desaparecía un dato fundamental del verbo en náhuatl: la valencia y transitividad del verbo (Launey, 1997: 21 y ss.).

\footnotetext{
${ }^{8}$ Es imprescindible consultar Hernández (2016: 11-59) en relación con el paradigma franciscano y sus avances lingüísticos.

${ }^{9}$ A los lectores actuales tal vez se nos escape que un 4 de mayo (de 1515) el Papa León X sancionó la bula De super impressione librorum, que prohibía la impresión de libros que no hubieran sido aprobados por la Inquisición, para dar una solución definitiva a los problemas de la imprenta con la Iglesia. Puede consultarse en $<$ http:// reader.digitale-sammlungen.de/de/fs1/object/display/bsb10152822_00008.html> [31.07.2018].
} 
Para Olmos la solución estaba clara. Los prefijos empleados usualmente con el verbo debían conservarse. Esta decisión plantea varios efectos en el caso de un verbo transitivo, a causa de la variedad de objetos definidos e indefinidos que puede tener:

1. Que un verbo que admita un objeto directo indefinido de persona o cosa, o un prefijo definido reflexivo sea incluido en el vocabulario con diferentes entradas. Por ejemplo, esto se aprecia en el caso de los verbos pachoa y nepanoa. Así el vocabulario de Olmos recoge la entrada "nitlapachoa: apretar, apesgar", " $n i-$ nopachoa: abajarse, ynclinarse" y "nitepachoa: regir o ser señor", y también "nitlanepanoa: juntar vno con otro" y "ninonepanoa: juntarse los casados" donde el tipo de objeto condiciona la asignación de un significado derivado que se desprende de su aplicación de uso, pero no de su significado primario, respectivamente, "aplastar" y "emparejar".

2. Que un verbo transitivo se incluya por un lado con un prefijo indefinido y por otro con un prefijo definido, considerándose voces independientes. Por ejemplo: "ninocaua: callar", "nitlacaua: sobrar", y "ni[c] caua: dexar algo de la mano y al ca[bo] de obra", correspondiente al verbo cahua ("cesar, dejar, abandonar").

3. Que un verbo cuya acción no sea realizada por una persona, se incorpora duplicado con un diferente sujeto. Es el caso por ejemplo de $t z a ̀ t z i$, que Olmos recoge en tres entradas primero como "nitzatzi: dar bozes" que vuelve a incluir como "nitzatzi: de a bozes" y luego "tzatzi: balar la obeja". ${ }^{10}$

Las consecuencias de este deseo de conservar las marcas de la valencia del verbo son evidentes. En primer lugar, la ordenación alfabética por la inicial del verbo es inútil, pues todos los verbos empezarían por ni-, nite-, nitla- o nic-/niqui-, lo que haría farragosa la ordenación y posterior búsqueda del verbo.

\footnotetext{
${ }^{10}$ A diferencia del español, donde balar, cacarear, mugir, etc. requieren un sujeto [+animal], en el náhuatl la marca [+humano] no es relevante en los sujetos del verbo $t z a ̀ t z i$, lo que es extensible a casos semejantes. Es notable que se apreciara este hecho y que se intentara reflejar en la misma lematización. Olmos pudo haberlo obviado, pero consideraba necesario aislar el significado derivado.
} 
Olmos cree que una solución es la clasificación de los verbos por su desinencia. Así los agrupa como verbos acabados en -huia, -tia, -ti, -li, -tla, -lia, -ma, -mi, -mia, -na, -ni, -nia, -oa/-ua, -ui, -çal-tza, -tzi, -ca, -tzo, -cha/chi, -chia, -pa, -pi, -pia, -qui, -quia, -qua, -ya. Baste decir que esta clasificación tiene poca utilidad aunque sea una estrategia de acercamiento comprensivo al tema del verbo que no llegó a cristalizar en ningún análisis de estas terminaciones como sufijos verbales y posibles indicadores semánticos.

La solución, por tanto, para Molina estaba clara: con el distanciamiento de fray Andrés de Olmos, podía analizar el verbo como un sustantivo o un adverbio, con el cuidado de informar sobre la valencia del verbo:

Los verbos activos en esta lengua, nunca se pronuncian ni hallan absolutos como en el romance dezimos amar, enseñar, oyr, \&c. sino siempre acompañados con pronombres o particulas que denoten la persona que padece y la que haze, assi como nitetlaçotla, nitemachtia, nitlacaqui. \&c. y porque poniendolos como ellos se pronuncian y usan con las tales particulas, fuera ymposible llevar orden de vocabulario; y tampoco no todos entendieran qual era el cuerpo del verbo y quales las particulas que se le añaden, por tanto llevo en los verbos este orden, que primero pongo el cuerpo del mismo verbo, y luego el pronombre o la partícula que le pertenece, quitandosela de delante y poniendola tras el: y despues su romance, juntamente con su preterito (Aviso tercero, 1945).

Molina aprovechaba precisamente la característica del náhuatl de ser una lengua aglutinante para resolver el problema gramatical, definiendo una raíz verbal que no se expresa de forma aislada en el discurso. Además, esta solución permite seguir manteniendo la enunciación del verbo en tiempo presente y primera persona del singular como ya contemplaba también Olmos. Éste puede ser un ejemplo más del peso de la gramática clásica en el tratamiento del verbo (no empleando la tercera persona del singular que deja más clara la raíz del verbo), aunque en tiempo presente la raíz se muestra invariable en todos los núme- 
ros. Cabe señalar además la inclusión de la forma pretérita del verbo que permite conocer y reconocer el verbo a pesar de tener la alteración de la base verbal. ${ }^{11}$ De todos modos, sea cual fuera el criterio final, el resultado permite comprender la indexación personal del verbo. Con el prefijo sujeto y los prefijos objeto definidos e indefinidos, justifica esta enunciación, a la que añadió el sentido del verbo (si es intransitivo, transitivo o bitransitivo; si el tipo de acción recae sobre cosas, animales o personas; si se emplean partículas o locativos, etc.).

No sólo se logra incluir los verbos en una ordenación alfabética, sino que dota a su vocabulario de una destacada capacidad didáctica (al mostrar el comportamiento del verbo al incorporarse los prefijos verbales) y la modificación de la raíz verbal, al expresar el tiempo perfectivo. En el caso de los verbos que cita en su aviso, estos aparecen como:

(1) Tlaçotla. nite. amar a otro. Preterito. onitetlaçotlac. [VMC]

(2) Machtia. nite. enseñar o predicar. p. onitemachti. [VMC]

(3) Caqui. nitla. oyr, entender, o escuchar. Preterito. onitlacac. [VMC]

Con esta lematización cualquier iniciado en la lengua náhuatl que sepa aislar o reconstruir la raíz verbal (en el caso de una forma pretérita, por ejemplo) puede localizarla como lema para conocer su significado y aprender su comportamiento verbal. En lo que respecta a la ordenación de las entradas, la ventaja es evidente, pues aunque en el caso de los verbos transitivos se siga otorgando a cada posible empleo con los prefijos objetos indefinidos y reflexivos una entrada propia, estos obviamente aparecerían consecutivos. De este modo, aunque no se desarrolle

\footnotetext{
${ }^{11}$ Pedro de Alcalá hace algo parecido en el caso del verbo en árabe. Reconoce el absurdo de establecer las conjugaciones a partir de las terminaciones, pues "Que tantas son las conjugaciones quantas en los verbos las terminaciones. Lo qual es muy superfluo de oyr" (1505: f. 10) y considera la enunciación del verbo en su vocabulario a partir de la primera persona del presente, mostrando a continuación la forma pretérita perfecta simple e imperativa para reconocer el verbo con la transformación de sus terminaciones.
} 
un sistema de acepciones propio de los diccionarios modernos, al menos permite su examen y comparación contigua. En el caso de los verbos intransitivos, en cambio, todas las definiciones se funden, generándose entonces sí una jerarquía de acepciones. Así ocurre con el ejemplo ya mencionado del verbo tzàtzi, en el que todas las voces antes citadas que aparecen dispersas en el vocabulario de Olmos, son incluidas por Molina en su VMC bajo una sola entrada: "Tzatzi. ni. Pregonar, dar bozes, balar la oueja, bramar el toro, o cantar el gallo de Castilla. P. otzatzic”. Así, la pertinencia de que el sujeto sea animal o persona en relación con el enunciado del lema no es preocupante, pues se desprende que se deriva de un significado primario más amplio que en castellano.

\subsection{Aspectos grafemáticos y de la pronunciación del náhuatl}

La ordenación alfabética requería una ortografía estandarizada, con un elenco preciso de grafemas, que se había desarrollado ya hacia 1530, si bien hubo diferencias entre los autores (Díaz y Bustamante, 1986). Molina había avanzado en la ortografía estandarizada ya en el VCM55. Si se tiene cuenta el VCM71, el franciscano había logrado culminar en el VMC una obra coherente en el plano de la ortografía. Sistematizó alfabéticamente el léxico, sin ninguna clasificación semántica añadida, atento a la codificación, lematización y definición de las voces. Molina quería aplicar para la lengua náhuatl, en este sentido, lo ya hecho por Antonio de Nebrija con la lengua latina y castellana, tal como nos explica en su aviso segundo del VMC:

Todos los vocablos Mexicanos se han de buscar en este Vocabulario, assi como los latinos y de romance se buscan en el Vocabulario del Antonio, por el mismo orden del Abece, conviene a saber, C. ante a. C. ante e. C. ante h. C. ante i. \&c. Y el mismo orden se guardara en las terceras y quartas letras que después desta es se siguen.

En este punto Molina debe abordar el problema de la escritura alfabética de la lengua náhuatl y la estandarización de la misma, de forma que se pueda aplicar este criterio de orde- 
nación de los lemas. El principal obstáculo es precisamente el marcado por la fonología que hace que este ordenamiento alfabético aparezca a los ojos de los españoles como "falto de letras". Así se ve obligado a explicar esa ausencia de letras en el orden alfabético como una anomalía que responde al hecho lingüístico de las no semejanzas del náhuatl con el castellano. En el aviso primero del VMC deja claro el orden que va a seguir a partir de esta contingencia:

Esta lengua Mexicana (como consta a todos los que bien sienten) carece de algunas letras de nuestro Abece, que son las siguientes. b. d. f. g. r. s. y a esta causa en el orden alphabetico deste Vocabulario, no se hallaran las sobredichas letras: sino que despues de la $\mathrm{A}$, se sigue la C, y tras ella la E, y luego la H I. L. M. N. O. P. Q. T. V. X. porque la Y griega, tampoco la ha menester esta lengua, pues le basta una. I. a lo menos para començar los vocablos en el orden que aqui se pone de las letras.

En este aspecto, Molina acaba reconociendo el peso de la particularidad de la fonología náhuatl frente a la latina o castellana, aunque no llega a llevar tal razonamiento al extremo de alterar tal orden alfabético como pretendía Olmos, según un criterio práctico que respetara más el modo de pronunciación del náhuatl que la forma de los grafemas elegidos para su representación, aunque fuera consciente de este hecho. De esta manera, de nuevo en el aviso segundo nos dice:

Por no tener esta lengua (como he dicho) todas las letras del Abece, me parecia que tampoco estava yo obligado a seguir totalmente el concierto que otros Vocabularios suelen llevar, de poner los vocablos arreo, yendo en todas las primeras letras dellos atado al orden del Abece, y ansi quise sacar deste estilo algunos vocablos, entendiendo que otro lugar les seria mas acommodado y adaptado, como era tras ça. poner ce. ci. ço. çu. y luego. cha. che. chi. cho. chu. Y assi mismo, porque el . tza. tze. tzi. tzo. tzu. (que se usa mucho en esta lengua) va a la pronunciacion de. ça. ce. ci. ço. çu. quiselo poner junto con el en la letra c. mas por parecerme que era lo mas acertado 
entrexerirlos todos, como aca uno le cupiesse su lugar, guardando el orden usado del Abece. y poner tambien el. tza. tze. tzi. tzo. tzu. en la letra T. (pues que se escribe con ella) aunque se pronuncie a manera de. ça. ce. ci. ço. çu. por tanto lo hize assi.

La intención inicial de incluir tza dentro de ça en la ordenación alfabética no es una propuesta original de Molina. Ya el mismo Olmos la tiene en cuenta, pues considera en su vocabulario los verbos acabados en -ça y -tza en el mismo grupo, hasta el punto de posiblemente considerarlos alófonos pues llega a confundir el verbo nitequequetza con el verbo nitequequeça. A este respecto se advierte que los primeros frailes gramáticos, y sobre todo Olmos, estaban muy atentos en la cuestión de la pronunciación y su representación grafemática como se comprueba en el capítulo $6^{\circ}$ de la ortographia del Arte de Olmos. En ese sentido Olmos presta más atención al hecho fonológico, mientras que Molina, en su Arte de la lengua mexicana y castellana (1571), obra en la que desarrolla y sistematiza de un modo más extenso el Arte de Olmos, se centra más en la prosodia de las palabras ( $2^{\mathrm{a}}$ parte, capítulo $9^{\circ}$, del buen sonido y del acento y buena pronunciacion).

Con este razonamiento, el peso del criterio literario sobre el lingüístico, hace que el reconocimiento de una fonología diferenciada solo se aplique a la representación y creación de dígrafos como grafemas, pero sin la consideración de signos fonemáticos. En el caso del fonema /i/ e /y/ Molina no los considera distintos, por lo que las palabras que comienzan en $y$ - están integradas en la letra $i-{ }^{12}$ Se reconoce que la pronunciación del náhuatl necesita un modo de representación que puede perfectamente fijarse con los recursos existentes adaptando letras y creando otras mediante dígrafos; de este modo, el franciscano mantiene lo hecho por otros frailes en el caso de la representación de la dental central sonora $/ \lambda /$ como $t l$ y de la palatal afri-

\footnotetext{
${ }^{12}$ En todos los ejemplos que mencionamos consideramos sólo el caso de las letras iniciales, a principio de palabra o raíz. No consideramos su representación como letra interna o final, pues plantea cuestiones que no afectan a la cuestión de la ordenación alfabética.
} 
cada sorda /č/ como ch. Se hace patente en estos casos el peso de la tradición escrituraria latina y española que impide la creación de "letras propias" que dificultarían, a ojos de los frailes, el aprendizaje y escritura de la lengua náhuatl, ${ }^{13}$ pero que también impiden, en algunas ocasiones, la creación de tipos estándar. Por ejemplo, la representación del fonema labiovelar $/ \mathrm{k}^{\mathrm{w}} / \mathrm{se}$ hace con los dígrafos $c u$ ante $e$ y $q u$ ante $a$, aplicando criterio castellano al primero y latino al segundo de modo que suene [ku]. El hecho de que no se mantenga qu ante $e$ es porque en castellano tiene valor de ke lo que dificultaría al lector castellano distinguir el sonido que representa, y el no usar $c u$ ante $a$ porque los propios frailes se dan cuenta de que el fonema $/ \mathrm{k}^{\mathrm{w}} /$ no suena igual a $[\mathrm{ku}]$.

En el caso de la mencionada dentoalveolar africada sorda / ts/, más cercana, como bien escucha Molina, a la sibilante sorda dental /s/ que a la dental /t/; opta por el dígrafo $t z$ frente a la $t c ̧$ que había pretendido imponer fray Andrés de Olmos, seguramente por presentarse esta opción como más útil para una ordenación alfabética, además de la simplificación de la escritura, algo complicada en Olmos por su afán fonológico. En cuanto a la sibilante sorda dental /s/ opta Molina por confirmar el uso de las letras ç y $c$ al emplearse estas ya en el castellano para representar el fonema fricativo sordo /s/. Y para la palatal fricativa sorda /š/, opta por la $x$, huyendo del uso de la $s$ tanto en el anterior caso como en este. Estos son solo algunos ejemplos de consonantes en los que se aprecia el intento de Molina de sistematizar la transcripción de los vocablos para favorecer su ordenación dentro de un esfuerzo colectivo por crear una ortografía normalizada que se desarrolló durante la segunda mitad del siglo XVI (cf. Smith Stark, 1995-1996: 408-409).

El resultado de todo ello es la creación de un alfabeto que no pretende ser fonético, pero establece una norma ortográfica necesaria para el diccionario y que permitiría desarrollar una literatura en lengua náhuatl. Se asienta así un criterio de escri-

\footnotetext{
${ }^{13}$ En esto hacemos notar un criterio similar en el aprendizaje de lenguas con escritura alfabética como el caso ya mencionado del árabe, en el que se ha empleado una trasliteración al alfabeto latino del árabe.
} 
tura que persigue clarificar la morfología y sintaxis del náhuatl. Así, por ejemplo, Molina no incluyó la entrada de Olmos nitemiquicelehuia como un verbo, sino que lo incluyó como una perífrasis verbal: miquiz eleuia. nino. La decisión no solo hace más comprensible la forma con un fin didáctico, sino que normativiza su escritura sin considerarla una composición verbal. De ese modo, búsqueda y comprensión se dan la mano en el uso del Vocabulario, pues "no basta saber la lengua, como quiera, sino entender bien la propriedad de los vocablos y maneras de hablar que tienen" (VCM71, Prólogo al lector).

\section{Conclusiones}

Esta búsqueda de la comprensión nos lleva a otra cuestión de interés, que no se dio en el caso de Pedro de Alcalá: hubo un notable esfuerzo por entender y hacer entendible aquello que no tenía una equivalencia precisa, con la finalidad de incluir el mayor caudal de léxico posible. Fray Pedro de Alcalá advertía que su Vocabulista no aspiraba a reunir todo el vocabulario de la lengua, pues la correspondencia entre lenguas no es exacta: "Non niego yo Reverenda Señoria auer algunos nonbres y avn muchos en la lengua arauiga los quales non se pusieron aquí, como non se ponen todos en los otros vocabulistas assi de latin como de romance, empero digo que los aquí puestos bastan para conplida y ordenadamente hablar la lengua arauiga [...]. Dexe algunas de las que alli estauan que carecian de traslación arauiga y si la tienen no assi vsitada, y por ello no assi inteligible" (Arte para ligeramente, 1505, prólogo). Aquí subyacen dos grandes problemas: a) las dificultades de querer incorporar en un vocabulario el mayor número de voces; $\mathrm{y}$ b) la obsesión por buscar equivalencias exactas entre las palabras de dos lenguas tan distintas.

En el VCM55 se apreciaba que fray Alonso de Molina quería ir más lejos que fray Andrés de Olmos, cuya ambición le llevó a recoger textos representativos de niveles sociolingüísticos populares y aristocráticos. Olmos se propuso incluir "todos los verbos o casi todos lo que ay en la lengua” (Arte, f. 225). Cuan- 
do en 1555 Molina forzó el castellano para dotar de una equivalencia a un vocablo náhuatl, ${ }^{14}$ lo hacía con el fin de que ese vocablo tuviera una entrada con vistas a su proyectado Vocabulario mexicano-castellano. De ahí la inclusión de neologismos, castellanismos y partículas. Todos los avisos del VCM55 hacen relación a la comprensión de los vocablos en náhuatl, pero también anuncian el modo en que se van a disponer las entradas en náhuatl del diccionario inverso (el VMC). Este afán justificaba la inclusión de frases o paráfrasis, como declara el aviso quinto del VMC: "si se ponen en este vocabulario algunas noticias o sentencias enteras, porque aunque esto parezca exceder los terminos de vocabulario, se tuuo mas en cuenta con que estas tales maneras de hablar son muy necesarias de saber, y dificultosas de componer, por esta misma razon se ponen tambien algunos verbos reuerenciales". Igualmente se pudieron incluir todos los numerales, lo que en el VCM55 y VCM71 era difícil de lograr por ser de base veinte el sistema de numeración náhuatl, con uso de cuantificadores y numerales concretos. De este modo se lograba una obra de una gran utilidad no solo para el estudiante, sino para el traductor.

Este afán justifica la inclusión de frases o paráfrasis. Como declara en su aviso quinto del VMC: "si se ponen en este vocabulario algunas noticias o sentencias enteras, porque aunque esto parezca exceder los terminos de vocabulario, se tuuo mas en cuenta con que estas tales maneras de hablar son muy necesarias de saber, y dificultosas de componer: por esta misma razon se ponen tambien algunos verbos reuerenciales". Igualmente se pudo incluir todos los numerales, cosa que en el VCM55 y VCM71 era difícil de lograr al ser el sistema de numeración náhuatl un sistema de base veinte y con uso de cuantificadores y numerales concretos. De este modo se logra una obra de una gran utilidad no solo para el estudiante, sino para un traductor

\footnotetext{
14 "En este vocabulario se ponen algunos romances, que en nuestro castellano no cuadran, ni se vsan mucho: y esto se haze por dar a entender mejor la propiedad de la lengua de los indios, y assi dezimos: Abaxador aunque no se vsa en nuestro romance, por declarar lo que quiere dezir esta palabra, tlatemouiani, la qual en buen romance quiere decir, el que abaxa algo" (VCM55, Aviso primero).
} 
que complemente su consulta con el resto de obras paralelas elaboradas por sus colaboradores y coetáneos.

El resultado es que tanto el VCM55 como VCM71 son la culminación de un vocabulario castellano-mexicano pero también el camino para lograr hacer viable el ansiado y retrasado vocabulario mexicano-castellano (VMC) que tan necesario resultaba. Que el fin del proyecto lexicográfico franciscano era este vocabulario que comenzaba en náhuatl lo confirma el aviso sexto: "los romances de los vocablos de la lengua (para que mejor se entienda la significación dellos) se han de buscar en este vocabulario que comienza en la lengua mexicana, por quanto en el estan mejor declarados que en el que comienza en romance, y la razon desto es porque se pudo mejor declarar cada vocablo de la lengua en particular, en el ya dicho vocabulario segundo que en el primero".

El acopio de léxico, resuelta la cuestión de cómo iba a ser tratada y expuesta la información lematizada, fue imparable. Colaboró fray Bernardino de Sahagún en el engrose del corpus desde primera hora. El tezcocano Hernando de Ribas aportó voces y definiciones para vocablos propios de diferentes registros y estilos de habla. La lematización de los vocablos tuvo que conciliar la eficacia en la búsqueda de un vocablo con las particulares características morfosintácticas del náhuatl como lengua aglutinante. Los sustantivos no ofrecían problemas, pero planteaban dificultades pragmáticas. Esto se hace extensible al caso de los verbos, pero aquí fue una cuestión más lexicográfica sobre el mejor modo de enunciar los vocablos tanto para su ordenación como para su comprensión por el lector de la obra. De esta manera, Molina "da informes completos: explicaciones y determinaciones locales y temporales, lo que, en la mayoría de los casos, hace posible comprender el contexto social y cultural, determinando, además, el tiempo prehispánico [...]. Se encuentran indicaciones de uso, y esto más allá de un simple registro de las unidades de un vocabulario [...]. Hay explicaciones detalladas que permiten comprender muchísimos detalles de la cultura ajena, reflejada en estas frases y unidades fraseológicas" (Thiemer-Sachse, 1997, 149). 
Con un vocabulario sin orden alfabético y sin una unificación de campos resultaba difícil no sólo la búsqueda, sino también saber qué es lo que no está en los registros o no se conoce. Es interesante contemplar en esta lucha por compilar el vocabulario de la lengua náhuatl el reconocimiento por parte de Molina de hallarse ante una batalla perdida. El lexicógrafo se hallaba ante una "mina (a manera de decir) inacauable de vocablos y maneras de hablar" y con el paso de los años volvía a recopilar infinidad de "otros vocablos de nuevo (de los muy muchos que quedan y quedaran siempre por poner)" (1571, Prólogo al lector). A pesar del esfuerzo, Molina reconocía la limitación de su trabajo, que los fransciscanos deberían de continuar: "enmendando lo que aquí va mal puesto, quitando lo superfluo, y añadiendo lo mucho que falta” (1571, Prólogo). No hubo descanso para el franciscano en Tlatelolco ni en Tetzcoco. Necesitaba entender a los nahuas porque quería convertirlos a la nueva fe mediante la evangelización en la lengua materna de los hablantes. Hubo que empezar por los cimientos: aprender y codificar el náhuatl para enseñárselo a los misioneros de lengua materna castellana que iban a evangelizar las nuevas poblaciones.

Desde el punto de vista evangelizador, la conquista novohispana se convertía en una extensión cronológica y geográfica de la expansión peninsular. Las estrategias lingüísticas y la situación de frontera tenían el precedente cercano y el desarrollo paralelo en la conquista de Granada como demuestran las obras publicadas: Arte para ligeramente saber la lengua arábiga, de Pedro de Alcalá (1505); la Doctrina Christiana en lengua Arauiga y Castellana, de Martín Pérez de Ayala (1504-1566) y la Recopilación de algunos nombres arábigos que los árabes pusieron a algunas ciudades y a otras muchas cosas, de Diego de Guadix (c. 1580). En consecuencia, el plan de actuación franciscana alcanzó tal envergadura que precisaba de las más perfectas herramientas lingüísticas. Hasta 1571 fray Alonso no pudo ver impresos en letra de molde su Arte y su Vocabulario náhuatl, pero su trayectoria nos permite conocer los desarrollos del paradigma franciscano en Tlatelolco y en México, a la sombra de 
los maestros Bernardino de Sahagún y Andrés de Olmos, en la estela americana de Elio Antonio de Nebrija.

\section{Bibliografía}

Alcalá, Pedro de (1505), Arte para ligeramente saber la lengua arauiga: Vocabulista arauigo en letra castellana, Granada, en <http://bdh-rd.bne.es/viewer.vm?id=0000037465>.

Díaz Rubio, Elena y Jesús Bustamante García (1986), "La alfabetización de la lengua náhuatl”, en A. Quilis y H.-J. Niederehe (eds.), The History of Linguistics in Spain, Ámsterdam / Filadelfia, John Benjamins, pp. 189-211.

Galeote, Manuel (en prensa), "A vueltas con las entradas religiosas en los vocabularios de Alonso de Molina (15551571)".

Galeote, Manuel y Miguel Figueroa-SaAvedra (2010), "Sobre la macroestructura del Vocabulario náhuatl (1555) que comienza en español”, en M. C. Ayala Castro y A. M. Medina Guerra (coords.), Diversidad lingüística y diccionario, Málaga, Universidad de Málaga, pp. 207-219.

Guadix, Diego De (2005 [c. 1580]), Recopilación de algunos nombres arábigos que los árabes pusieron a algunas ciudades y a otras muchas cosas, edición de Elena Bajo Pérez y Felipe Maíllo Salgado, Gijón, Trea.

Hernández, Ascensión (2014), "Ensayo introductorio”, en A. de Nebrija, Gramática sobre la lengua castellana, edición de Carmen Lozano, México, Academia Mexicana de la Lengua, pp. IX-XLV.

Hernández, Ascensión (2016), “Tradiciones, paradigmas y escuelas. Una visión general de las gramáticas misioneras mesoamericanas", Historiographia Linguistica, 43:1-2, pp. 11-59.

Hernández, Ascensión y Miguel León Portilla (1993), "Estudio introductorio y trasliteración", en Arte de la lengua mexicana, de F. Andrés de Olmos, Madrid, Ediciones de Cultura Hispánica, Instituto de Cooperación Iberoamericana. 
Hernández, Ascensión y Miguel León-Portilla (2009), Las primeras gramáticas del Nuevo Mundo, México, Fondo de Cultura Económica.

Lafaye, Jacques (2002), Albores de la imprenta. El libro en España y Portugal y sus posesiones de ultramar (siglos XV y XVI), México, Fondo de Cultura Económica.

Launey, Michel (1997), "La elaboración de los conceptos de la diátesis en las primeras gramáticas del náhuatl”, en K. Zimmermann (ed.), La descripción de las lenguas amerindias en la época colonial, Fráncfort / Madrid, Vervuert / Iberoamericana, pp. 21-41.

León Portilla, Miguel (1977), "Estudio preliminar”, en Vocabulario en lengua castellana y mexicana, de Fr. Alonso de Molina, México, Porrúa.

Máynez, Pilar (2002), El Calepino de Sahagún: un acercamiento, México, Universidad Nacional Autónoma de México / Fondo de Cultura Económica.

Mendieta, Jerónimo de (1973), Historia eclesiástica indiana, 2 vols., Madrid, Ediciones Atlas (Biblioteca de Autores Españoles).

Molina, Alonso de (1555), Aquí comiença vn vocabulario en la lengua castellana y mexicana, México, Juan Pablos, edición facsimilar con "Estudio preliminar" de Manuel Galeote, Anejos 37 de Analecta Malacitana, Málaga, Universidad de Málaga, 2001 (se cita como VCM55).

Molina, Alonso de (1565), Confessionario mayor, en lenguamexicana y castellana, México, Antonio de Espinosa Impresor.

Molina, Alonso de (1571), Arte de la lengua mexicana y castellana, México, Casa de Pedro Ocharte.

Molina, Alonso de (1571), Vocabvlario en lengva castellana y mexicana, México, Casa de Antonio de Spinosa [Facsímil, Madrid, Ediciones de Cultura Hispánica, 1945] (se cita como VCM71).

Molina, Alonso de (1571), Vocabvlario en lengva mexicana y castellana, México, Casa de Antonio de Spinosa [Facsímil, Madrid, Ediciones de Cultura Hispánica, 1945] (se cita como VMC). 
Montes de Oca Vega, Mercedes (2013), Los difrasismos en el náhuatl del siglo XVI y XVII, en <http://www.iifilologicas. unam.mx/ebooks/los-difrasismos-en-el-nahuatl/mobile/ index.html\#p=649> [consultado el 22 de septiembre de 2018].

Montes de Oca Vega, Mercedes (2016), “Más allá de la nahuatlización del cristianismo", en E. Hernández y P. Máynez (eds.), El Colegio de Tlatelolco: síntesis de historias, lenguas y culturas, México, Grupo Destiempos, pp. 242-262.

Olmos, Andrés de (1985), Arte de la lengua mexicana y vocabulario, edición de René Acuña, introducción, advertencias, paleografía y apéndices de Thelma D. Sullivan, México, Universidad Nacional Autónoma de México.

Olmos, Andrés de (1990 [1553]), Tratado de hechicerías y sortilegios, edición de Georges Baudot, México, Universidad Nacional Autónoma de México / Centro de Estudios Mexicanos y Centroamericanos.

Olmos, Andrés de (1993 [1547]), Arte de la lengua mexicana (edición facsimilar), introducción y transliteración de Ascensión Hernández y Miguel León Portilla, Madrid, Ediciones de Cultura Hispánica, Instituto de Cooperación Iberoamericana.

Pérez de Ayala, Martín (1980 [1504-1566]), Doctrina Christiana, en lengua Arauiga, y Castellana: compvesta e impressa por mandado del Illustrissimo y Reverendissimo Señor don Martin de Ayala, Arçobispo de Valencia: para la instruction de los nueuamente conuertidos deste Reyno, Valencia, Ioan Mey.

Pezzi, Elena (1989), El vocabulario de Pedro de Alcalá, Almería, Cajal.

Ringmacher, Manfred (1997), "El Vocabulario náhuatl de Molina leído por Humboldt y Buschmann”, en K. Zimmermann (ed.), La descripción de las lenguas amerindias en la época colonial, Fráncfort / Madrid, Vervuert / Ibero-americana, pp.75-112.

Romero RANGel, LAUra (2013), “Innovaciones lexicográficas del primer diccionario de la Nueva España: el Vocabulario 
castellano-mexicano (1555) de Alonso de Molina”, Revista Argentina de Historiografía Lingüística, V:1, pp. 41-62. Siméon, RÉmi (1977 [1885]), Diccionario de la lengua náhuatlo mexicana, México, Siglo XXI.

Smith Stark, Thomas C. (1995-1996), "La grafía náhuatl de Antonio del Rincón”, Revista Latina de Pensamiento y Lenguaje, 2:2, pp. 407-433.

Stols, Alexander A. M. (1989 [1962]), Antonio de Espinosa: el segundo impresor mexicano, México, Universidad Nacional Autónoma de México.

Thiemer-Sachse, Úrsula (1997), "El Vocabulario castellanozapoteco y el Arte en lengua zapoteca de Juan de Córdova -intenciones y resultados (perspectiva antropológica)", en K. Zimmermann (ed.), La descripción de las lenguas amerindias en la época colonial, Fráncfort / Madrid, Vervuert / Iberoamericana, pp. 147-174.

\section{Manuel Galeote}

Licenciado en Filosofía y Letras (1986) y doctor en Filología Hispánica por la Universidad de Granada, con una tesis doctoral sobre el léxico indoamericano en los textos de los cronistas andaluces de las Indias Occidentales (1993), ejerce la docencia en la Universidad de Málaga desde 1996. En la Universidad de Berna (Suiza) ocupó la Cátedra extraordinaria de Lingüística Hispánica. Impartió docencia en las Universidades de Neuchâtel y Friburgo (2004-2007). Fue el primer director y el fundador de la "Sección de Lingüística Hispánica" (Institut für Spanische Sprache und Literaturen de la Universidad de Berna) entre 2004 y 2007. Ha realizado estancias de investigación en El Colegio de México, Universidad de Costa Rica y Universidad Metropolitana de Ciencias de la Educación (UMCE de Santiago de Chile). Ha impartido conferencias e intervenido en congresos nacionales e internacionales de Europa y América. Sus investigaciones se han centrado en las hablas andalu- 
zas, el costumbrismo lingüístico, la Lingüística misionera y la Historia de la lengua española entre Andalucía y América, con especial atención en los últimos años a cuestiones históricodialectales de la terminología de la oleicultura.

Ha editado (Granada, 1990 y Málaga, 2001) los estudios de Dialectología andaluza de J. Mondéjar (Universidad de Granada), en colaboración con la catedrática Pilar Carrasco Cantos de la Universidad de Málaga. Asimismo, publicó en 2010, en colaboración con A. López-Bernasocchi, su asistente en la Universidad de Berna, el volumen Tesoro castellano del primer diccionario de América: lemas y concordancias del vocabulario español-nahuatl (1555) de Alonso de Molina (Verbum, Colección Mayor, 685 pp.). Dentro de la colección Hispanica Helvetica (Académie Suisse des Sciences Humaines et Sociales, Sociedad Suiza de Estudios Hispánicos, Universidad de Lausana y Pórtico Librerias), publicó en 2007 un estudio extenso y una reproducción facsimilar (por primera vez en libro) del extenso artículo "Voces andaluzas (o usadas por autores andaluces) que faltan en el Diccionario de la Academia Española", que vio la luz en Francia en la Revue Hispanique (1920, 116, pp. 313-647). Han visto ya la luz tres volúmenes de la Obra selecta de C. de Castro (ed. y coord. de M. Galeote), que publica la Diputación Provincial de Córdoba y el Ayuntamiento de Iznájar (Córdoba): Novelas cordobesas (2010), Textos feministas (2011) y Novelas de Rusia (2017, incluye estudios de Lily Litvak, Antonio Cruz y Juana Toledano). Su edición facsimilar con un estudio preliminar del primer Vocabulario castellanonáhuatl (México, 1555) de fray Alonso de Molina se publicó en 2001 en los Anejos de la Revista de Filología Analecta Malacitana (Universidad de Málaga). En colaboración con el experto nahuatlato Miguel Figueroa Saavedra (Universidad Veracruzana), tiene dispuesta para la imprenta la nueva edición de este primer vocabulario impreso en América.

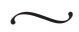

\section{Miguel Figueroa-SAavedra}

Es profesor-investigador del Instituto de Investigaciones en Educación de la Universidad Veracruzana y miembro del 
Sistema Nacional de Investigadores nivel 1. Realizó estudios en la Universidad Complutense de Madrid, de Licenciatura en Antropología de América y de Doctorado en Antropología Social. Ha sido profesor en el Museo de América de Madrid, España, del Instituto de Salud Pública y del departamento de lenguas de la Universidad Veracruzana Intercultural en la Universidad Veracruzana, impartiendo experiencias sobre lengua y literatura náhuatl, comunicación en salud, terminología y lexicografía, y políticas del lenguaje. Actualmente participa como docente en la Maestría y Doctorado en Investigación Educativa del Instituto de Investigaciones en Educación, y la Maestriah ipan Totlahtol iwan Tonemilis de la Universidad Veracruzana Intercultural. Sus líneas de investigación son lengua y cultura náhuatl, planificación y normalización lingüística de las lenguas nacionales en la educación superior, e historiografía lingüística y lexicografía. 